\title{
Facility Layout Planning of Central Kitchen in Food Service Industry: Application to the Real-Scale Problem
}

\author{
Nobutada Fujii, Toshiya Kaihara, Minami Uemura, \\ Tomomi Nonaka, and Takeshi Shimmura \\ Graduate School of System Informatics, Kobe University \\ Rokkodai 1-1, Nada, Kobe 657-8501, Japan \\ \{nfujii,nonaka\}@phoenix.kobe-u.ac.jp, kaihara@kobe-u.ac.jp, \\ uemura@kaede.cs.kobe-u.ac.jp, t-shinmura@gankofood.co.jp
}

\begin{abstract}
Central kitchen is a food producing factory to improve productivity in the food-service industry, to pursue scale-merit by aggregation of tasks in multiple stores into one place. However, the central kitchen is still labor intensive production environment because of tradeoff between quality and quantity of products, so that the handicraft of skilled workers cannot be eliminated. In addition, there are many parttime workers as well as full-time ones, as a result, operators can be also uncertain factors. Furthermore, customer demand forecast is also difficult according to not only susceptible to weather and seasonal variation but also influence from irregular events held around the restaurant. Due to such characteristics of the food service industry, it is also difficult to plan proper facility layout of the central kitchen to achieve both optimality and adaptability to the complexity. In this study, a new facility layout planning method of the central kitchen is proposed, where not only optimization but simulation is also adopted; flow of workers as well as products can be considered simultaneously. Computer experiments in which the proposed method is applied to the real-scale problem are conducted to confirm the effectiveness of the proposed method.
\end{abstract}

Keywords: Food service industry, Facility layout, Simulation, Genetic Algorithm.

\section{Introduction}

Importance of the service industry is increasing because the service industry plays about $70 \%$ of GDP in Japan, two-thirds of employment. In spite of the expansion of the role of the service industry, the productivity growth of the service industry is relatively low compared to the manufacturing industry and the overseas service industry [1]. It is not easy to improve the productivity of the service industry because service is complex in nature, so that the quality of production and provision of services depends on experience and intuition of workers. Service Science [2], Product-Service System [3] and Service Engineering [4 have been proposed to improve productivity of service industry.

V. Prabhu, M. Taisch, and D. Kiritsis (Eds.): APMS 2013, Part II, IFIP AICT 415, pp. 33-40, 2013.

(C) IFIP International Federation for Information Processing 2013 
Productivity of food service industry is also relatively low among the service industries [56]. Central kitchen is a food processing plant in order to improve productivity in the food service industry, to pursue economies of scale. In the central kitchen, efficiency of production due to the automation and process integration is important, however, according to trade-off between quality improvement and quantity acquirement the handicraft of skilled workers cannot be eliminated; the production base labor intensive. In addition, there are many part-time workers as well as full-time ones; unskilled workers may be uncertain factors in production. Moreover, not only variables of central kitchen inside, it is subject to weather and seasonal variation. There is also the impact of irregular events held around the restaurant, so that demand forecasting is intensely difficult. By producing food of high quality while adapting flexibly to the environmental changes of central kitchen internal and external, it is possible to achieve improvement customer satisfaction (CS), employee satisfaction (ES) and management satisfaction (MS).

From the above-mentioned complexities, daily productions of the current central kitchen is carried out by the experience and intuition of skilled workers. Consequently, facility layout does not take into account the movement of workers as well as product flow. Moreover, the production is labor intensive because it is hand-made to create value, so that the load on the worker is large, reduction of the workload by improving the facility layout is also necessary. This study aims at deriving facility layout to minimize the moving mileage of workers as the worker load indicator. Flow line of worker draws a complicated trajectory, calculating the length of moving mileage is difficult by the conventional mathematical programming approach [7]. Therefore, to calculate workers' moving mileage, integration of computer simulations and genetic algorithms is proposed [8]. Fitness calculation of each individual in GA incorporates the computer simulations, as a result, the placement of equipment and workers are obtained to minimize the sum of moving mileage of each worker. However, it is necessary a lot of computation time if experiments with sufficient number of generations are performed assuming evaluate the layout only by the simulation, because the simulations are executed (number of individuals) $\times$ (number of generations) times.

Therefore, simple calculation method of worker's mileage by accumulating mileage of workers along each product flow is introduced, so that a sufficient number of generations can be performed in GA. Subsequently, further GA with computer simulations is performed. The proposed integration method is applied to a real-scale problem in this paper.

\section{Integration of Simulation and Genetic Algorithm}

\subsection{Algorithm of the Proposed Method}

The proposed method consists of two stages; simple calculation of worker's mileage is done and the sum of obtained values is used as fitness value of GA in the first stage. In the second stage followed, including some of the solutions 
obtained in the first stage as initial population, the GA is conducted with calculating fitness value by performing simulations. Perform a sufficient number of generations GA minutes faster in the first stage, then it can create a layout that takes into account both the flow of workers and products further in the second stage. The flowchart of the proposed method is shown in Fig. 1

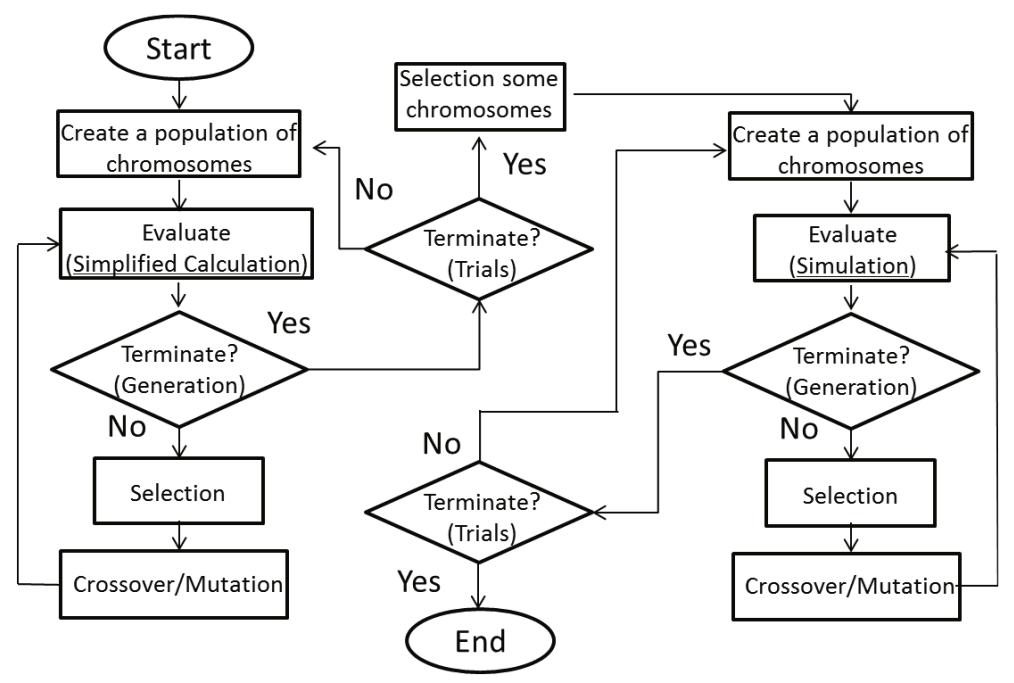

Fig. 1. Algorithm of the proposed method

\subsection{Calculation of Workers' Moving Mileage}

Simplified calculation of the workers' moving mileage is determined as follows along to each product. The fitness of the GA is calculated by sum of each worker's mileage with respect to all products.

- Mileage between facilities is calculated based on the center coordinates of the facility.

- Worker who performs the next process receives the product at the facility, delivers it to the following facility, processes it and returns to the waiting area.

- Worker who is responsible for the last process is to convey to OUTPUT.

- Alternative facilities and workers are assigned in random manner.

When the operator acts as the rules above, as Fig. 2, a triangle is composed by the flow line of workers among waiting area of the worker, facility of the process and the following facility. Furthermore, since the worker was in charge 


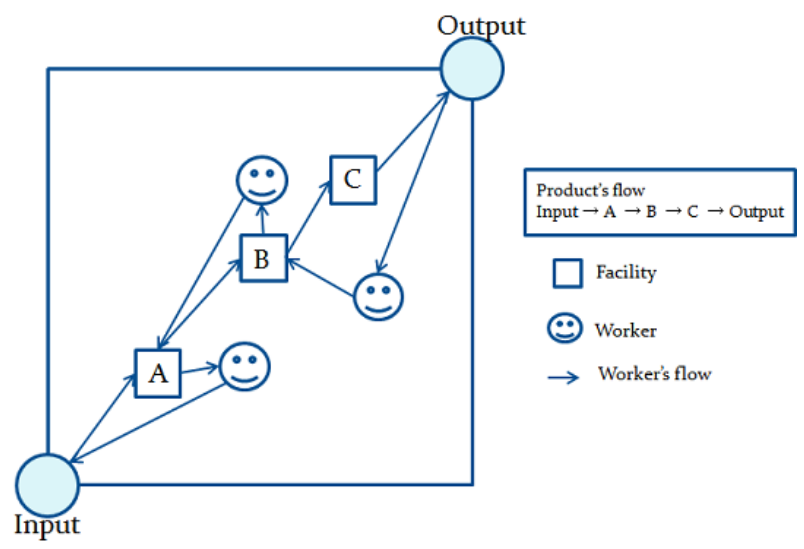

Fig. 2. Calculation method of workers' moving mileage

of the process is transported to the OUTPUT, rectangle as shown in the figure is formed in the final step. The workers' moving mileage are calculated by summing each product minutes triangles and quadrilaterals thereof.

\subsection{Coding and Genetic Operation}

Each individual in the GA represents the position information of each facility. It is assumed that expressed in numeric position of the partition that discretizing the manufacturing area, separated location information. Fig. 3 depicts an

Discretized manufacturing area

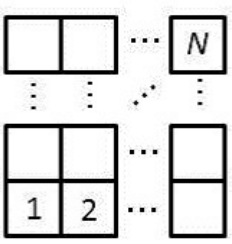

Facility $B$ is 36

:

Bit length is equivalent to the number of facilities

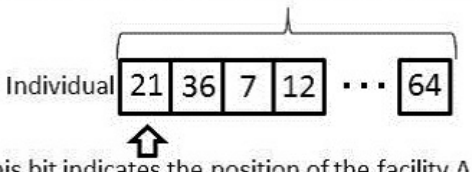

Fig. 3. coding method 


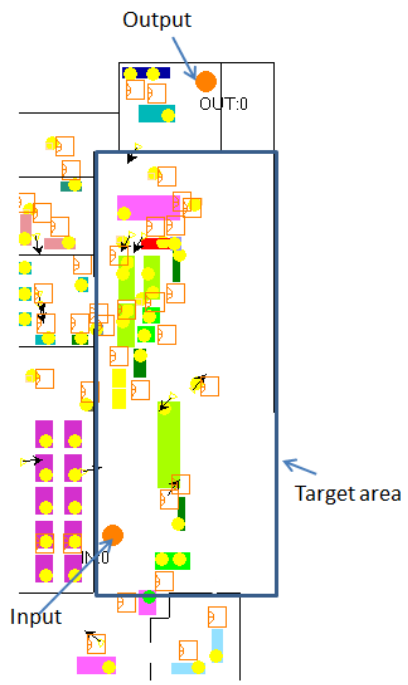

Fig. 4. Current layout of the central kitchen

overview of the coding; each locus represents the position of the corresponding machine in the discretized production floor.

To prevent the facility and worker of two or more are present in one compartment separated, generation of individual, crossover and mutation operation are executed to avoid duplication in each bit of one individual.

- Generation of initial individuals: determine the position number in each individual from the bit head, but avoid duplicate numbers by going to random selection from the set excluding the numbers used in the previous bits.

- Crossover: the two-point crossover is used. Duplicated bits are changed to the random number that does not overlap with other bits.

- Mutation: select at random from the number that does not overlap with any bits itself has.

\section{Experimental Results and Discussion}

\subsection{Experimental Conditions}

Fig. 4 illustrates an overview of the central kitchen to target. The target area represents the area of interest of the facility layout plan in this study. Each product is dispatched from the Input location, and is collected at the Output location which is a shipping place. All facility number of plant is 55 units and the worker is 14 , however, target area has facilities and workers to act as the planning target, 23 units, five operators, respectively. The target area is separated into 8 $\times 16$ rectangular areas where each facility and waiting area of worker is allocated 
Table 1. Sum of workers mileage in the busy season

\begin{tabular}{|c|c|c|c|}
\hline BEST $(\mathrm{m})$ & Ave. $(\mathrm{m})$ & S.D. & Time $(\mathrm{sec})$ \\
\hline 22167.90 & 23581.71 & 919.89 & 113365 \\
\hline
\end{tabular}

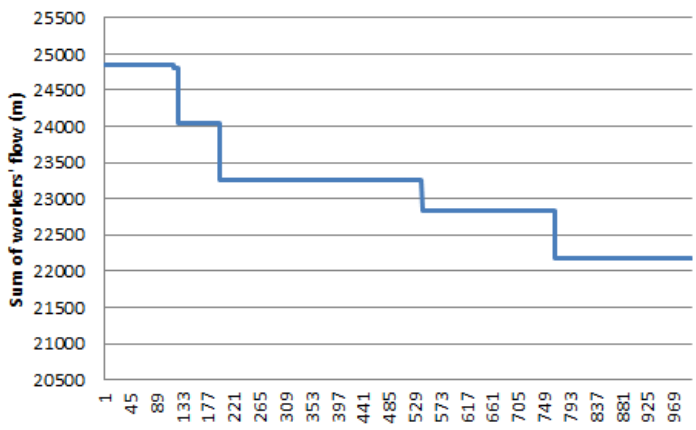

Fig. 5. Transition of mileage in the 2nd step

to one rectangle so as to achieve minimizing sum of workers' moving distance. Product varieties are set to 22 .

GA uses different parameters in the first and second stages from the viewpoint of the balance of the calculation time. Each parameter setting is as follows.

- 1st stage: population 1000, generation 20000, crossover rate 0.6 , mutation rate 0.01

- 2nd stage: population 100 , generation 1000 , crossover rate 0.6 , mutation rate 0.01

The tournament selection with tournament size 2 and elite selection strategy are also adopted.

\subsection{Results and Discussion}

100 trials execute in the first stage, subsequently, 10 trials run in the second stage which include 10 best layouts obtained in the first stage as initial individuals. Table 1 represents the resultant best, mean and standard deviation value of obtained workers' moving mileage and the average computation time after the execution of the second stage. In addition, Fig. 5 represents the transition of the workers' mileage of attempts best solution is obtained.

As shown in the figure, compared with the layout obtained in the first stage, the value can be about 2,000 $\mathrm{m}$ shortened from $24,800 \mathrm{~m}$. The result reveals that further combination of GA and simulation can obtain a better layout taking into account the flow of product and operators based on the layout determined by GA using a simplified calculation of fitness value. 


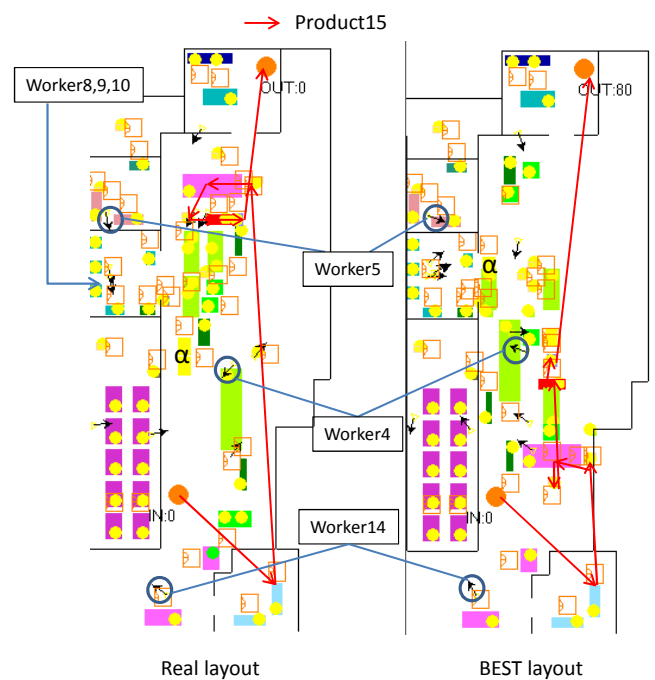

Fig. 6. Current and proposed layout

Table 2. Comparison with the current layout

\begin{tabular}{|c|c|c|}
\hline & Current & Proposed \\
\hline Worker1 $(\mathrm{m})$ & 1145.83 & 1510.83 \\
\hline Worker2 $(\mathrm{m})$ & 1408.99 & 1772.96 \\
\hline Worker3 $(\mathrm{m})$ & 1646.48 & 1621.69 \\
\hline Worker4 $(\mathrm{m})$ & 1909.62 & 1452.51 \\
\hline Worker5 $(\mathrm{m})$ & 1425.84 & 1128.33 \\
\hline Worker6 $(\mathrm{m})$ & 1858.01 & 1436.33 \\
\hline Worker7 (m) & 1809.20 & 1622.52 \\
\hline Worker8 $(\mathrm{m})$ & 1283.34 & 1374.17 \\
\hline Worker9 $(\mathrm{m})$ & 884.78 & 976.473 \\
\hline Worker10 $(\mathrm{m})$ & 1366.49 & 679.557 \\
\hline Worker11 (m) & 1507.92 & 1346.49 \\
\hline Worker12 (m) & 3107.61 & 3000.16 \\
\hline Worker13 (m) & 663.82 & 663.82 \\
\hline Worker14 $(\mathrm{m})$ & 4272.79 & 3582.59 \\
\hline Sum $(\mathrm{m})$ & 24303.39 & 22167.90 \\
\hline
\end{tabular}

Comparison between the obtained layout and the current layout in the central kitchen is done. Table 2 shows moving mileage of each worker in both layouts. Fig. 6] also indicates the layout obtained by the proposed method (BEST) and the real central kitchen layout (Real). The standby position of each worker to be associated with the product flow of Product 15 is also illustrated in each layout. Looking at the flow diagram, after dispatched from the Input location, Product 
15 is processed by Worker 14 below the layout. Transport distance is extended when Worker 14 delivers the product to the next step in the current layout; it requires only transport up to the center in the facility layout of BEST, but the facility of the next process is located above in the current layout.

It can be understood as the difference of transportation during this period has become a difference of Worker 14 between two layouts. It also seems that Worker 4 can obtain shorter distance in the obtained layout because Worker 4 is allocated to near position to the room in the left hand side of the layout where Worker 8, 9 and 10 are arranged; Worker 4 comes and goes to the room frequently during production.

\section{Conclusion}

This paper presented a new facility layout planning method of the central kitchen in food service industry by combining optimization, GA, and simulation; fitness value calculation was done by simplified numerical method in the first stage, then the simulation was also conducted in the second stage to consider both product and operator flow. Comparison results with the current layout of the central kitchen revealed the effectiveness of the proposed method.

Elaboration of simulation and combination with the shift-scheduling of workers can be pointed out as the further steps of the study.

This research is partly supported by Service Science, Solutions and Foundation Integrated Research Program ( $\mathrm{S}^{3}$ FIRE), RISTEX/JST.

\section{References}

1. Ministry of Economy, Trade and Industry: Towards Innovation and Productivity Improvement in Service Industries (2007), http://www.meti.go.jp/english/report/data/0707SPRING.html

2. Spohrer, J., Maglio, P.P., Bailey, J., Gruhl, D.: Steps toward a science of service system. Computer 40(1), 71-77 (2007)

3. Mont, O.K.: Clarifying the Concept of Product-Service System. Journal of Cleaner Production 10(3), 237-245 (2002)

4. Shimomura, Y., Tomiyama, T.: Service Modeling for Service Engineering. In: IFIP International Federation for Information Processing, vol. 167, pp. 31-38 (2005)

5. Takenaka, T., Shimmura, T., Ishigaki, T., Motomura, Y., Ohura, S.: Process management in restaurant service $-\mathrm{A}$ case study of Japanese restaurant chain. In: Proc. of International Symposium of Scheduling (ISS 2011), pp. 191-194 (2011)

6. Shimamura, T., Kaihara, T., Fujii, N., Takenaka, T.: Improving customer's subjective waiting time introducing digital signage. In: Emmanouilidis, C., Taisch, M., Kiritsis, D. (eds.) APMS 2012, Part II. IFIP AICT, vol. 398, pp. 385-391. Springer, Heidelberg (2013)

7. Kusiak, A., Heragu, S.S.: The facility layout problem. European Journal of Operations Research 29, 229-251 (1987)

8. Fujii, N., Kaihara, T., Uemura, M., Shimmura, T.: A Study on Sales-production Integrated Service Manufacturing Systems - Layout Planning of Central Kitchen in a Food-service Industry. In: Proc. of Design Symposium 2012, pp. 241-244 (2012) (in Japanese) 